\title{
Liquid Impact on a Permeable Solid Body
}

\author{
Yuriy A. Semenov ${ }^{\mathrm{a}}$, Guo Xiong $\mathrm{Wu}^{\mathrm{a}}$ \\ ${ }^{a}$ Department of Mechanical Engineering, University College London, Torrington Place, \\ London WC1E 7JE, UK
}

\begin{abstract}
The free surface flow and the hydrodynamic loads generated by impact between a liquid wedge and a permeable solid body are investigated. The study is carried out within the framework of self-similar solution, which is realistic for this kind of configuration and over the short period of impact. We study the effect of liquid penetration through the porous/perforated solid surface on the pressure distribution and flow pattern. An integral hodograph method is employed to convert the differential equation in the fluid domain into integral equations along the axes of a parameter plane, from which the problem corresponding to the impermeable solid surface is a special case. The system of integral equations are solved numerically using the method of successive approximations. The results are presented for streamline patterns, pressure distribution along the solid surface of permeable wedges.

Key words: Liquid/solid impact; Permeable body ; Self-similar flow; Integral hodograph method.
\end{abstract}

\section{Introduction}

Liquid/structure impact is a widely observed natural phenomenon. Examples include wave impacts on marine structures and coastline, slamming of ships, landing of aircrafts on a water surface, motion of planing crafts and droplet impact on aircrafts. High speed liquid impacts can generate very high loading

Email addresses: semenov@a-teleport.com (Yuriy A. Semenov), g.wu@ucl.ac.uk (Guo Xiong $\mathrm{Wu}$ )

Preprint submitted to Elsevier

June 18, 2015 
on a solid body that may cause structural damage or failure. Perforated and porous solid structures have been proposed as a way to reduce hydrodynamic loading during wave impacts [1]. A perforated body allows liquid penetration though its surface, which decreases the hydrodynamic pressure. Examples of perforated structures include wave breakers and "stabilizer" of the Roseau tower which consists of an open-ended square box attached to the frame of the tower subjected to wave impacts. Another example of a perforated structure is a tubular frame, usually used as a protection for subsea installations on the seabed. Arrays of renewable energy devises or their elements in some cases also can be considered as a perforated structure for its interaction with incoming waves. Review of practical applications of perforated structures in offshore and coastal engineering as well as methods predicting hydrodynamic forces was presented by Molin [1]. In order to understand better the fluid structure interaction of the processes mentioned above and provide an effective way to predict the wave loadings, it is necessary to account for permeability of the structure in the wave impact processes.

Impacts of interest usually lasts for a very short period of time, during which the pressure and fluid velocity vary rapidly both with time and in space. In general, the process is fully transient and the temporal and spatial variables are fully independent. One way to solve this kind of problem is based on the Wanger's theory together with the technique of matched asymptotic expansions, especially in the context of marine applications $[2,3,4]$. However, in many cases, especially at initial stage and in some local areas, the flow may be treated as self similar. Moreover, at this stage the hydrodynamic force may reach its maxima value that is important to estimate safety and reliability at the design stage.

In the studies mentioned above, the bodies in the impact are usually considered as rigid and impermeable. However there are publications, notably those by Molin and Korobkin [5], Iafrati and Korobkin [6], and Iafrati et al. [7], in which the effect of permeability on the hydrodynamic force during wave impacts is investigated. In this study we will obtain analytical/numerical solution for self similar flow to study an effect of permeability of a solid structure on hy- 
drodynamic loads which are important for assessing safety and reliability of the structure. The Integral hodograph method $[8,9]$ which is an extension of the classical hodograph method is applied. The method enables the original partial differential equation with nonlinear boundary conditions to be converted into a set of integral equations along the straight lines, which are then solved numerically. It has been successfully used in variety of water impact problems $[8,10,11]$. However the application of the method to the present problem has some new major challenges. On the impermeable solid surface, the normal velocity is unknown a priori and depends on the pressure which has to be determined from the solution. The boundary condition is given in terms of a relationship between the pressure and the normal component of the velocity trough the body surface which is satisfied under iteration procedure.

Various case studies related to the permeability of the solid body are presented. Both the solid body and the wave front are considered in the form of a wedge of various angles. It includes the cases of impact of the wave crest on to the perforated wall as well as water entry of the perforated wedge into half - space of the liquid. The terms porous and perforated solid surfaces are used to distinguish the difference in the boundary condition for penetration of the liquid through the body surface. In the first one a linear relationship between the pressure and normal velocity through non deforming body boundary is employed, that corresponds to a porous body when the diameter of holes are much smaller that its length and the holes are finely distributed in the body [6]. In the second case, a perforated body is considered, for which the quadratic relationship between the pressure and the normal velocity trough the non deforming body surface is used [5]. These two laws of the liquid penetration are related to the applications in coastal and offshore engineering $[1,5,12]$ where a massive of wave breakers or a cover subsea installations, forms the porous/perforated structure, used to reduce the fluid impact on a structure.

To outline briefly, the present study generalizes the previous integral form of solution $[8,10]$ for liquid impacting onto impermeable rigid bodies to permeable ones. Section 2 describes the mathematical formulation and the solution proce- 
a)

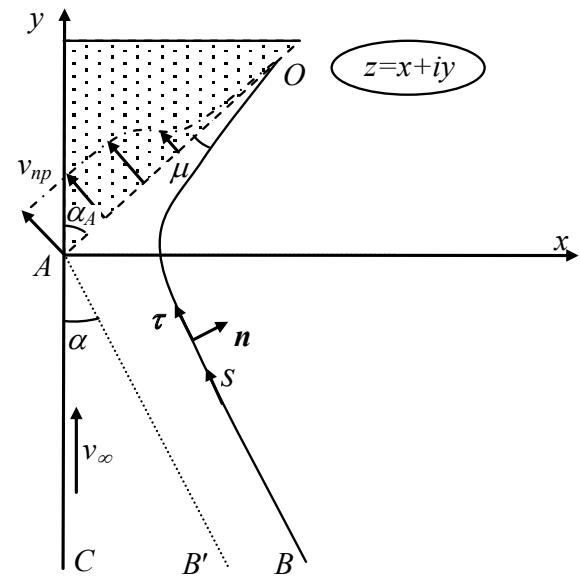

b)

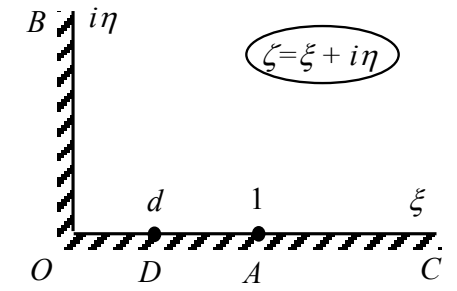

Figure 1: Sketch of the problem for impact between a liquid wedge (dotted line at the time of impact) and an permeable solid wedge: $(a)$ similarity plane and $(b)$ parameter plane.

dure based on the integral hodograph method which reduces the problem to a system of integral equations. The numerical results, their analysis and physic implications based on the flow patterns, pressure distribution and free surface, as well as the flux into the permeable body are discussed in Section 3.

\section{Formulation of the problem and the solution procedure}

We consider the impact problem between a liquid and solid permeable wedge of half-angle $\alpha$ and $\alpha_{A}$, respectively. The liquid is assumed to be ideal and incompressible, the flow to be irrotational, and the incoming velocity is constant. The gravity and surface tension effects are ignored. The flow is self similar and will be studied in the frame of reference with its origin attached to the solid wedge. A sketch of the problem is shown in figure 1.

The Cartesian coordinate system $x y$ with origin at point $A$ is defined with $y$ axis along the line of the flow symmetry. The liquid wedge has uniform velocity $V$ along the $y$-axis, which is marked as $v_{\infty}$ in the similarity plane in figure $11 a$. 
The fluid particle at point $A$ when $t=0$ coincides with point $O$. After impact, a jet with a tip of angle $\mu$ moves to point $O$ along the permeable wedge side. The symbol $v_{n}$ in the figure is the normal velocity due to body surface permeability, and it tends to zero at the contact point $O$, where the pressure tends to ambient pressure.

For a constant impact velocity of the liquid wedge, the time-dependent problem in the physical complex plane $Z=X+i Y$ can be written in the stationary similarity plane $z=x+i y$ in terms of the self-similar variables $x=X /(V t)$, $y=Y /(V t)$, where $t$ is time. As a result the inflow velocity at infinity $v_{\infty}=1$ at points $B$ and $C$ in figure $1 a$. The complex velocity potential $W(Z, t)$ for the self-similar flow can be written as

$$
W(Z, t)=V^{2} t w(z)=V^{2} t[\phi(x, y)+i \psi(x, y)]
$$

The problem is to determine the function $w(z)$ which conformally maps the similarity plane $z$ onto the complex potential region $\mathrm{w}$. We choose the first quadrant of the plane in figure $1 b$ as the parameter region to derive expressions for the non dimensional complex velocity, $d w / d z$, and for the derivative of the complex potential, $d w / d \zeta$, both as functions of the variable $\zeta$. Once these functions are found, the velocity field and the relation between the parameter region and the physical flow region can be determined as follows:

$$
v_{x}-i v_{y}=\frac{d w}{d z}(\zeta), \quad z(\zeta)=z(0)+\int_{1}^{\zeta} \frac{d w}{d \zeta^{\prime}} / \frac{d w}{d z} d \zeta^{\prime},
$$

where $v_{x}$ and $v_{y}$ are the $x$ - and $y$-components of the velocity nondimensionalized by $V$.

Conformal mapping allows us to fix three arbitrary points in the parameter region, which are chosen $O, B$ (a point at infinity) and $A$ as shown in figure 1b. In this plane, the positive part of the imaginary axis $(0<\eta<\infty, \xi=0)$ corresponds to the free surface $O B$. The interval $(0<\xi<1, \eta=0)$ of the real axis corresponds to the wetted part of the wedge, and the rest of the positive real axis $(1<\xi<\infty, \eta=0)$ corresponds to the symmetry line $A C$. The point $\zeta=1$ in figure $1 b$ is the image of the point $A$ from the similarity plane $\zeta$. 
2.1. Expressions for the complex velocity and the derivative of the complex potential, $d w / d \zeta$

. When the body surface is an impermeable rigid, the normal component of the velocity relative to the body surface equals zero, and therefore the velocity on the body surface is directed along its tangential direction. In the present problem we consider a permeable body surface, where the normal component of the velocity is nonzero and the direction of the velocity on the body surface becomes unknown. It presents an additional challenge to the solution procedure.

The boundary-value problem for the complex velocity function is formulated in the parameter plane. At this stage we introduce function $\beta(\xi)=$ $-\arg (d w / d z)$ along the interface $O A$, i.e. on the interval $0<\xi<1$ of the real axis of the parameter plane, and function $v(\eta)$ which is the velocity modulus along the free surface $O B$, or along the positive part of the imaginary axis of the $\zeta$ - plane. With these notations, we have

$$
\begin{aligned}
& \chi(\xi)=\arg (d w / d z)=\left\{\begin{array}{lcc}
-\beta(\xi), & 0<\xi<1, & \eta=0, \\
-\pi / 2, & 1<\xi<\infty, & \eta=0 .
\end{array}\right. \\
& v(\eta)=\left|\frac{d w}{d z}\right|, \quad 0<\eta<\infty, \quad \xi=0 .
\end{aligned}
$$

When we approach point $A$ along the interface $O A$, the velocity direction $\beta(\xi)=\tan ^{-1}\left(v_{y} / v_{x}\right)_{\xi \rightarrow 1-\varepsilon}, \varepsilon \rightarrow 0$, tends to the value $\pi / 2$, or coincides with the direction of the $y$-axis, since $v_{x}(\xi)_{\xi \rightarrow 1-\varepsilon} \rightarrow 0$ due to the flow symmetry, and $v_{y}(\xi)_{\xi \rightarrow 1-\varepsilon}>0$ for any small permeability of the interface. For the impermeable interface there is a jump in the function $\beta(\xi)$ at point $\xi=1$, since the velocity direction coincides with the direction of the interface, or $\beta(\xi)=\tan ^{-1}\left(v_{y} / v_{x}\right)_{\xi \rightarrow 1-\varepsilon} \rightarrow \pi / 2-\alpha, \varepsilon \rightarrow 0$. The formulation of the problem corresponds to the permeable body surface, and the case of the impermeable body surface is a particular one for which the liquid flowrate through the body tends to zero. Thus, for both cases the function $\chi(\xi)$ in Eq.(3) is continuous function at point $A$ and along the whole real axis. 
We can confirm that the integral formula $[8,9]$ for $F(\zeta)=d w / d z$ below

$$
F(\zeta)=v_{\infty} \exp \left[\frac{1}{\pi} \int_{0}^{\infty} \frac{d \chi}{d \xi} \ln \left(\frac{\zeta+\xi}{\zeta-\xi}\right) d \xi-\frac{i}{\pi} \int_{0}^{\infty} \frac{d \ln v}{d \eta} \ln \left(\frac{\zeta-i \eta}{\zeta+i \eta}\right) d \eta+i \chi_{\infty}\right],
$$

satisfies the given conditions on the real and imaginary axes of the first quadrant in Eqs.(3) and (4), or: $\chi(\xi)=\arg [F(\zeta)]_{\zeta=\xi}, 0<\xi<\infty, \eta=0$, and $v(\eta)=$ $|F(\zeta)|_{\zeta=i \eta}, 0<\eta<\infty$, where $v_{\infty}=v(\eta)_{\eta \rightarrow \infty}, \chi_{\infty}=\chi(\xi)_{\xi \rightarrow \infty}$. Using the known value in the first line of Eq.(3), Eq.(5) becomes

$$
\frac{d w}{d z}=v_{0} \exp \left[\int_{0}^{1} \frac{d \beta}{d \xi} \ln \left(\frac{\xi-\zeta}{\xi+\zeta}\right) d \xi-\frac{i}{\pi} \int_{0}^{\infty} \frac{d \ln v}{d \eta} \ln \left(\frac{i \eta-\zeta}{i \eta+\zeta}\right) d \eta-i \beta_{0}\right] .
$$

where $v_{0}=v(\eta)_{\eta=0}$ and $\beta_{0}=\beta(\xi)_{\xi=0}=\pi / 2-\alpha_{A}$ are the velocity magnitude and direction at point $A$. The functions $\beta(\xi)$ and $v(\eta)$ will be determined later from the free surface kinematic and dynamic boundary conditions.

In order to obtain expression for the derivative of the complex potential in the parameter plane, $d w / d \zeta$, it is useful to introduce the unit vectors $\vec{n}$ and $\vec{\tau}$ on the fluid boundary, which are normal and tangent to the surface, respectively. The former is directed outward from the liquid region, and while moving in the latter direction along the boundary, the spatial coordinate $s$ increases and the liquid region is on the left hand side (see figure $1 a$ ). With this notation, we have

$$
d w=\left(v_{s}+i v_{n}\right) d s
$$

where $v_{s}$ and $v_{n}$ are the tangential and normal velocity components along the flow boundary, respectively. Let $\theta(\eta)=\tan ^{-1}\left(v_{n} / v_{s}\right)$ and $\gamma(\xi)=\tan ^{-1}\left(v_{n} / v_{s}\right)$ denote the angles between the velocity vector with $\vec{\tau}$ on the flow boundary. The former is defined along the imaginary axis of the parameter and therefore corresponds to the free surface $O B$ in the similarity plane, while the latter is on the real axis and corresponds to the interface and the symmetry line. Eq.(7) allows us to determine the argument of the derivative of the complex potential, 

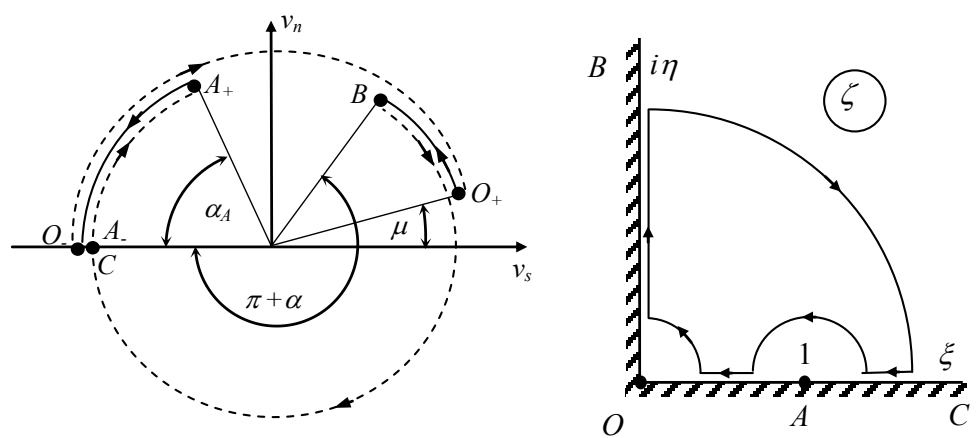

Figure 2: (a) Behaviour of the velocity angle to the flow boundary, $\tan ^{-1}\left(v_{n} / v_{s}\right)$ : the solid lines for the continuous changes while the dashed lines for the step changes. (b) The corresponding variation in the parameter region.

$\vartheta=\arg (d w / d \zeta)$

$\vartheta(\zeta)=\arg \left(\frac{d w}{d \zeta}\right)=\arg \left(\frac{d w}{d s}\right)+\arg \left(\frac{d s}{d \zeta}\right)=\left\{\begin{array}{l}\gamma(\xi), \quad 0<\xi<1, \quad \eta=0, \\ \theta(\eta)+\pi / 2, \xi=0,0<\eta<\infty .\end{array}\right.$

By analyzing the behaviour of the velocity angle along the whole flow boundary in figure 2 , we can see the variation of $\tan ^{-1}\left(v_{n} / v_{s}\right)$. It is continuous along the free surface $O B$ (defined as $\theta(\eta)$ ) and on the permeable surface $O A$ (defined as $\gamma(\xi)$ ) respectively, as shown by solid lines. The function $\theta(\eta)$ has step changes or discontinuities at points $O, B$ and $C$, while for the function $\gamma(\xi)$ the discontinuity occurs at point $A$. These step changes are shown by dashed lines in figure $2 a$. They may lead to singularities in the expression for the derivative of the complex potential.

Now we determine the functions $\theta(\eta)$ and $\gamma(\xi)$ along the fluid boundary on which they are defined, that is, along the positive parts of the imaginary and real axes of the $\zeta$-plane, respectively. Between points $C$ and $A_{-}, 1<\xi<\infty$, function $\gamma(\xi) \equiv-\pi$, since $v_{n}=0$ and $v_{s}<0$. When we move in counter clockwise direction along an infinitesimal semicircle centred at the point $\zeta=1$ in the parameter plane, the function $\gamma(\xi)=\tan ^{-1}$, changes from $-\pi$ at $A_{-}$ 
to $-\pi-\alpha_{A}$ at $A_{+}$. This is because velocity components $v_{x} \rightarrow 0$ and $v_{y}>0$, which gives $v_{s}=-v_{y} \cos \left(\alpha_{A}\right)$ and $v_{n}=v_{y} \sin \left(\alpha_{A}\right)$. Thus, the jump in the function $\gamma(\xi)$ at the point $A$ is $\Delta_{A}=-\alpha_{A}$. When we move from point $A_{+}$ to point $O_{-}$along the wedge surface, the function $\gamma(\xi)$ changes continuously from $\gamma_{A}=-\pi-\alpha$ to $\gamma_{0}=-p i$ at point $O_{-}$. When we move in counter clockwise direction along an infinitesimal quarter of the circle centred at the point $\zeta=0$ in the parameter plane the corresponding line in the physical plane passes through the tip $O$ of the tip jet. The jump in the function $\tan ^{-1}\left(v_{n} / v_{s}\right)$ equals $\Delta=\mu-\pi$ as it is seen from figure 2 , where $\mu=\theta(\eta)_{\eta}=0$. By taking into account Eq.(8) we can see that the jump in $\arg (d w / d \zeta)$ equals $\Delta_{\vartheta}=\Delta+\pi / 2=$ $\mu-\pi / 2$. The corresponding change of the $\operatorname{argument} \arg (\zeta)$ equals $\pi / 2$, and so we can expect that function $d w / d \zeta$ at point $O(\zeta=0)$ has a singularity of order $d w / d \zeta \zeta^{2 \Delta_{\vartheta} / \pi}$. When moving from point $O$ to point $B$ along the imaginary axis of the parameter plane, the function $\theta(\eta)$ changes continuously from value $\theta_{0}$ to the value $\theta_{B}=\theta(\eta)_{\eta \rightarrow \infty}$.

We can write function $\vartheta(\zeta)$ as follows

$$
\vartheta(\zeta)=\arg \left(\frac{d w}{d \zeta}\right)= \begin{cases}-\pi, & 1<\xi<\infty, \quad \eta=0, \\ -\pi+\Delta_{A}+\gamma(\xi), & 0<\xi<1, \quad \eta=0, \\ \theta(\eta)+\Delta_{\vartheta}, \quad \xi=0, & 0<\eta<\infty .\end{cases}
$$

The problem is then to find the function $d w / d \zeta$ in the first quadrant of the parameter plane which satisfies the boundary condition (9). This is a homogeneous boundary value problem, or $\arg (d w / d \zeta)$ is given on the entire boundary. It can then be confirmed that the function obtained from the following integral formula $[8,9]$

$$
G(\zeta)=K \exp \left[\frac{1}{\pi} \int_{\infty}^{0} \frac{d \vartheta}{d \xi} \ln \left(\zeta^{2}-\xi^{2}\right) d \xi+\frac{1}{\pi} \int_{0}^{\infty} \frac{d \vartheta}{d \eta} \ln \left(\zeta^{2}+\eta^{2}\right) d \eta+i \vartheta_{\infty}\right],
$$

where $K$ is a real factor, $\vartheta(\zeta)=\arg [G(\vartheta)], 0<\xi<\infty, \eta=0$ and $0<\eta<\infty$, $\xi=0, \vartheta_{\infty}=\vartheta(\zeta)_{|\zeta| \rightarrow \infty}$ can meet the condition in Eq.(9). By performing the integration in Eq.(10) over steps where the value $\vartheta(\zeta)$ is known, we finally obtain 
the expression for the derivative of the complex potential in the $\zeta$-plane as

$$
\frac{d w}{d \zeta}=K \frac{\zeta^{2 \mu / \pi-1}}{\left(1-\zeta^{2}\right)^{\alpha / \pi}} \exp \left[\frac{1}{\pi} \int_{0}^{1} \frac{d \gamma}{d \xi} \ln \left(\xi^{2}-\zeta^{2}\right) d \xi+\frac{1}{\pi} \int_{0}^{\infty} \frac{d \theta}{d \eta} \ln \left(\zeta^{2}+\eta^{2}\right) d \eta\right] .
$$

Integration of Eq.(11) in the parameter region allows us to obtain the function $w(\zeta)$ which conformally maps the parameter region onto the corresponding region in the complex potential plane:

$w(\zeta)=w_{A}+K \int_{1}^{\zeta} \frac{\zeta^{2 \mu / \pi-1}}{\left(1-\zeta^{2}\right)^{\alpha / \pi}} \exp \left[\frac{1}{\pi} \int_{0}^{1} \frac{d \gamma}{d \xi} \ln \left(\xi^{2}-\zeta^{2}\right) d \xi+\frac{1}{\pi} \int_{0}^{\infty} \frac{d \theta}{d \eta} \ln \left(\zeta^{2}+\eta^{2}\right) d \eta\right] d \zeta^{\prime}$,

where $w_{A}$ is the complex potential at point $\mathrm{A}$ and can be taken as zero without loss of the generality.

Dividing (11) by (6), we can obtain the derivative of the mapping function

$$
\begin{aligned}
\frac{d z}{d \zeta} & =\frac{K}{v_{0}} \frac{\zeta^{2 \mu / \pi-1}}{\left(1-\zeta^{2}\right)^{\alpha / \pi}} \exp \left[\frac{1}{\pi} \int_{0}^{1} \frac{d \gamma}{d \xi} \ln \left(\xi^{2}-\zeta^{2}\right) d \xi+\frac{1}{\pi} \int_{0}^{\infty} \frac{d \theta}{d \eta} \ln \left(\eta^{2}+\zeta^{2}\right) d \eta\right. \\
& \left.-\frac{1}{\pi} \int_{0}^{1} \frac{d \beta}{d \xi} \ln \left(\frac{\xi-\zeta}{\xi+\zeta}\right) d \xi+\frac{i}{\pi} \int_{0}^{\infty} \frac{d \ln v}{d \eta} \ln \left(\frac{\eta-\zeta}{\eta+\zeta}\right) d \eta+i \beta_{0}\right]
\end{aligned}
$$

The integration of this equation yields the mapping function $z=z(\zeta)$ relating the parameter and similarity planes.

We notice that in contrast to the impact between the liquid and impermeable solid wedges [10], the functions $\beta(\xi)$ and $\gamma(\xi)$ characterizing respectively the direction of the velocity vector with $x$-axis and the angle between the velocity vector and the permeable solid surface now become unknown on $A O$. The first function appears in the expression for the complex velocity in Eq.(6), and the second appears in the expression for the derivative of the complex potential in Eq.(11). In addition, at point $A$, or $\zeta=1, \gamma(\xi)$ is continuous for the impermeable surface but discontinuous for the permeable surface. The latter leads to an additional singularity in the expression for the derivative of the complex potential at point $A$ at, $\zeta=1$, as it can be seen in Eq.(11), which requires additional attention in the solution procedure. 
The governing equations (6) and (11) - (13) enable to determine the pressure coefficient at any point of the flow region. By choosing point $A$ as the reference point in the Bernoulli equation, where $w_{A}=0$, and $v_{A}=\left|d w / d z_{\zeta=1}\right|$, and taking the advantage of the self-similarity of the flow, we can determine the pressure coefficient at any point $z$ in the flow region through

$$
c_{p}^{*}=\frac{P-P_{A}}{\rho V^{2}}=\Re\left(-w+z \frac{d w}{d z}\right)-\frac{1}{2}\left|\frac{d w}{d z}\right|^{2}+\frac{1}{2} v_{A}^{2}
$$

where $P$ is the pressure at the point $z$ and $P_{A}$ is the pressure at the point $A$. Then, the pressure coefficient based on the ambient pressure, $P_{a}$, which can be represented by the pressure $P_{O}=P_{a}$ at point $O$, can be determined as follows

$$
c_{p}(\xi)=\frac{P-P_{a}}{\rho V^{2}}=c_{p}^{*}(\xi)-c_{p}^{*}(0)
$$

The governing equations (6) and (11) - (13) contain the unknown parameter $K$ and the functions $\gamma(\xi), \beta(\xi), \theta(\xi)$ and $v(\eta)$, all to be determined from physical considerations, as well as the dynamic and kinematic boundary conditions on the free surface and the permeable body surface.

At the moment of impact, the tip of the liquid wedge will move into the body surface due to permeability. At the same time it will also spread along the body surface, which will depart from the body surface at point $D$ and will eventually become point $O$. We notice that when the flow is self similar the velocity at point $O$ is constant. Thus the coordinate position of point $O$ can be decided by its velocity $V_{O}=V v_{0}$ relative to point $A$. This gives $Z_{O}=V t z_{O}=V_{0} t e^{i \beta_{0}}=V t v_{0} e^{i \beta_{0}}$, which further gives $z_{O}=V t v_{0} e^{i \beta_{0}}$, where $\left.v_{0}=v(\eta)\right)_{\eta=0}$ and $\beta_{0}=\beta(\xi)_{\xi=0}$. The same argument was used Semenov, Wu \& Oliver [11] for the liquid/liquid impact. From this, the distance between points $A$ and $O$ in the similarity plane equals $v_{0}$, which can be used to determine the parameter $K$ :

$$
K\left|\int_{0}^{1} \frac{1}{K} \frac{d z}{d \zeta}\right|_{\zeta=\xi} d \xi \mid=v_{0}
$$


2.2. Determination of the functions $\theta(\eta)$ and $v(\eta)$ on $O B$ from boundary conditions on the free surface.

The dynamic boundary conditions on the free surface $O B$ for an arbitrary self-similar flow can be derived in the following form [8], by exploiting the Bernoulli equation and the fact that the acceleration of a liquid particle is orthogonal to the free surface with constant pressure

$$
\begin{gathered}
\frac{d \theta}{d s}=\frac{v+s \cos \theta}{s \sin \theta} \frac{d \ln v}{d s}, \\
\frac{1}{\tan \theta} \frac{d \ln v}{d s}=\frac{d}{d s}\left[\arg \left(\frac{d w}{d z}\right)\right] .
\end{gathered}
$$

Multiplying both sides of Eqs.(17) and (18) by $d s / d \eta=|d z / d \zeta|_{\zeta=i \eta}$ we obtain the following integro-differential equation for the function $\theta(\eta)$ :

$$
\frac{d \theta}{d \eta}=\frac{v+s \cos \theta}{s \sin \theta} \frac{d \ln v}{d \eta},
$$

where $s=s(\eta)$ is obtained from integration of the expression $-|d z / d \zeta|_{\zeta=i \eta}$ along the imaginary axis of the parameter plane. Determining the argument of the complex velocity from Eq.(6) and substituting the result into Eq.(18), the following integral equation for the function $d(\ln v) / d \eta$ is obtained:

$$
-\frac{1}{\tan \theta} \frac{d \ln v}{d \eta}+\frac{1}{\pi} \int_{0}^{\infty} \frac{d \ln v}{d \eta^{\prime}} \frac{2 \eta^{\prime}}{\eta^{\prime 2}-\eta^{2}} d \eta^{\prime}=\frac{1}{1+\eta^{2}}+\frac{1}{\pi} \int_{0}^{\infty} \frac{d \beta}{d \xi} \frac{2 \xi}{\xi^{2}+\eta^{2}} d \xi .
$$

The system of equations (19) and (20) enables us to determine the functions $\theta(\eta)$ and $d(\ln v) / d \eta$ along the imaginary axis of the parameter domain. Then, the velocity magnitude on the free surface can be obtained from

$$
v(\eta)=v_{\infty} \exp \left(-\int_{\eta}^{\infty} \frac{d \ln v}{d \eta^{\prime}} d \eta^{\prime}\right)
$$

where $v_{\infty}=1$ is the reference velocity at infinity. This gives the velocity at point $O, v_{0}=v(\eta)_{\eta=0}$.

\subsection{Determination of functions $\gamma(\xi)$ and $\beta(\xi)$ from the kinematic boundary condition on the permeable solid surface.}

In the porous media, the velocity of the fluid flow is proportional to the pressure gradient, based on Darcy's law. When the solid becomes very thin 
with a pressure jump from one side of the surface to the other side, the velocity normal to the body is then proportional to the difference of pressures from both sides. In our case, at the initial stage of the impact, which is usually of main interest, we assume that the pressure on the back side of the solid surface remains to be ambient pressure $P_{a}$, therefore, the normal component of the velocity through the wetted surface can be written as :

$$
V_{n}=\alpha_{p}\left(P-P_{a}\right)
$$

where the coefficient $\alpha_{p}$ characterizes the porosity of the thin wall. When $\alpha_{p} \rightarrow$ $0, V_{n} \rightarrow 0$ in Eq.(22). This returns to the impermeable boundary condition.

On the surface of a perforated body, the relationship between the normal component of the velocity and the pressure can be written as $V_{n}\left|V_{n}\right|=\chi_{0}(P-$ $\left.P_{a}\right) / \rho$, as $V_{n}$ is expected to be positive $[1,5]$

$$
V_{n}=\chi_{0} \sqrt{\left(P-P_{a}\right) / \rho}, \quad \chi_{0}^{2}=\frac{2 \nu \kappa^{2}}{1-\kappa} .
$$

where $\nu$ is a discharge factor, and $\kappa$ is the ratio between the area of the holes and the total area, $\rho$ is the liquid density as defined previously. As $\kappa \rightarrow 0$, the impermeable boundary condition is recovered. In the following, non-dimensional parameters are used based on the definitions of $v_{n}=V_{n} / V, \alpha_{0}=\alpha_{p} \rho V$, while $\chi_{0}$ is already non-dimensional. Eqs.(22) and (22) can be respectively written as

$$
\begin{gathered}
v_{n}=\alpha_{0} c_{p}, \\
v_{n}=\chi_{0} \sqrt{c_{p}},
\end{gathered}
$$

The tangential component of the velocity on the wedge side $O A$ can be determined with the notation in Eq.(7) as

$$
v_{s}(\xi)=\Re\left(\frac{d w}{d z} \frac{d z}{d s}\right)=\Re\left(\left.\frac{d w}{d z}\right|_{\zeta=\xi} e^{i \delta_{A}}\right)
$$

where $\delta_{A}=-\pi-\alpha$ is the direction of the vector $\vec{\tau}$. Then, the function $\gamma(\xi)$ can be obtained

$$
\gamma(\xi)=\tan ^{-1}\left(\frac{v_{n}(\xi)}{\Re\left(e^{i \delta_{A}} d w / d z_{\zeta=\xi}\right)}\right)
$$


where the normal component of the velocity, $v_{n}$, will be detrmined in the following. Taking the argument of Eq.(13), we obtain $\delta_{A}=\beta+\gamma$, that gives equation for the function $\beta(\xi)$ as follows

$$
\beta(\xi)=\delta_{A}-\gamma(\xi)
$$

The integral equation (20) with Eq.(19) allows us to obtain the functions $\theta \eta$ and $v(\eta)$, together with the functions $\gamma(\xi)$ and $\beta(\xi)$ determined from Eqs.(27) and (28). Once these functions are found, the velocity at point $O, v_{0}$, and the angle of the tip of the splash jet, $\mu$, can also be found.

The numerical solution of the system of equations, which is strongly nonlinear, is based on the iteration with the relaxation coefficient used for the functions $\beta \xi$ and $\gamma(\xi)$ being $1 / 5$ of that for the functions $\theta(\eta)$ and $v(\eta)$. It starts with $\beta(\xi) \equiv=\beta_{0}, \gamma(\xi) \equiv=-\pi, v_{n}(\xi)=0$ on the wedge side $O A$ and $\theta(\eta)=\alpha$, $v(\eta)=1$ on free surface $O B$, as initial values. The unknown $K$ is obtained from Eq.(16) at each iteration. Eq.(15) gives pressure from which $v_{n}$ is determined from Eqs. (24) or (25), for porous and perforated bodies, respectively. New functions $\beta \xi$ and $\gamma(\xi)$ are then determined from Eq, (27) and Eq.(28), respectively. The functions $\theta(\eta)$ and $v(\eta)$ are determined from Eq.(19) and Eq.(20), respectively, which give the free surface shape $O B$. The iteration returns to Eq.(16) and the process is repeated until the convergence has been achieved.

\section{Numerical results}

\subsection{Numerical approach}

The numerical approach employed in the present study is based on the method of successive approximations, which is similar to that used by Semenov $\& \mathrm{Wu}$ [10], for solving self-similar impact problems between the impermeable solid and liquid wedges. Let us consider two sets of points distributed along the part of the real axis, $0<\xi_{j}<1, j=1 \ldots M$, and the imaginary axis, $0<\eta_{i}<\eta_{N}, j=1 \ldots$, where $\eta_{N}$ is sufficiently large. The integrals within each segment in the system of equations are evaluated explicitly, using the linear interpolation for the functions $\gamma(\xi), \beta(\xi), \theta(\eta)$ and $v(\eta)$, on the intervals 


\begin{tabular}{cccccccc}
$\alpha_{0}$ & \multicolumn{3}{c}{$\mu / \pi$} & \multicolumn{5}{c}{$c_{p \max }$} \\
& $\mathrm{N}=150$ & $\mathrm{~N}=300$ & $\mathrm{~N}=600$ & $\mathrm{~N}=150$ & $\mathrm{~N}=300$ & $\mathrm{~N}=600$ & $(\mathrm{I} \& \mathrm{~K})$ \\
\hline 0 & 0.00962 & 0.00985 & 0.00989 & 6.99 & 6.90 & 6.88 & 6.88 \\
0.1 & 0.00534 & 0.00562 & 0.00567 & 5.82 & 5.70 & 5.68 & 5.68 \\
0.2 & 0.00281 & 0.00310 & 0.00315 & 5.05 & 4.90 & 4.88 & 4.88 \\
0.3 & 0.00139 & 0.00165 & 0.00170 & 4.49 & 4.32 & 4.29 & 4.29 \\
0.4 & 0.00066 & 0.00085 & 0.00089 & 4.03 & 3.86 & 3.83 & 3.83 \\
0.5 & 0.00031 & 0.00043 & 0.00045 & 3.65 & 3.49 & 3.46 & 3.45
\end{tabular}

Table 1: Convergence study and comparison for water-entry of a porous solid wedge $\alpha_{A}=60^{\circ}$ into the half-space of the liquid $\left(\alpha=90^{\circ}\right)$.

$\left(\xi_{j-1}, \xi_{j}\right)$ and $\left(\eta_{i-1}, \eta_{i}\right)$, respectively. The results contain the unknowns coefficients $\Delta \gamma_{j}=\gamma_{j}-\gamma_{j-1}, \Delta \beta_{i}=\beta_{i}-\beta_{i-1}, \Delta \theta_{i}=\theta_{i}-\theta_{i-1}$ and $\ln \left(v_{i} / v_{i-1}\right)$, which are determined from the system of equations (19), (20), (27), (28).

\subsection{Impact between liquid and solid porous wedges.}

Effect of body porosity on the flow parameters including the slamming pressure peak has been studied using the boundary element method (BEM) by Iafraity \& Krobokin [6] for a porous wedge entering the flat water surface. Similar problem of a permeable block sliding along an inclined beach has been studied by Iafrati, Miloh \& Korobkin [7], in the context of violent free surface flow generated by landslide.

To evaluate the accuracy and mesh-independence of the results, several distributions with different numbers of nodes have been employed. Table 1 gives results with $N=150, N=300$ and 600 for a porous wedge of half-angle impacting the liquid wedge with $\alpha=90^{\circ}$, which is in fact a problem of a solid porous wedge entering a flat free surface. It can be seen from the table that the contact angle $\mu / \pi$ and the maximum pressure coefficient have converged well with the number of nodes. The converged pressure coefficients are virtually identical to those of Iafrati \& Korobkin [6] (I\&K) obtained through BEM. 

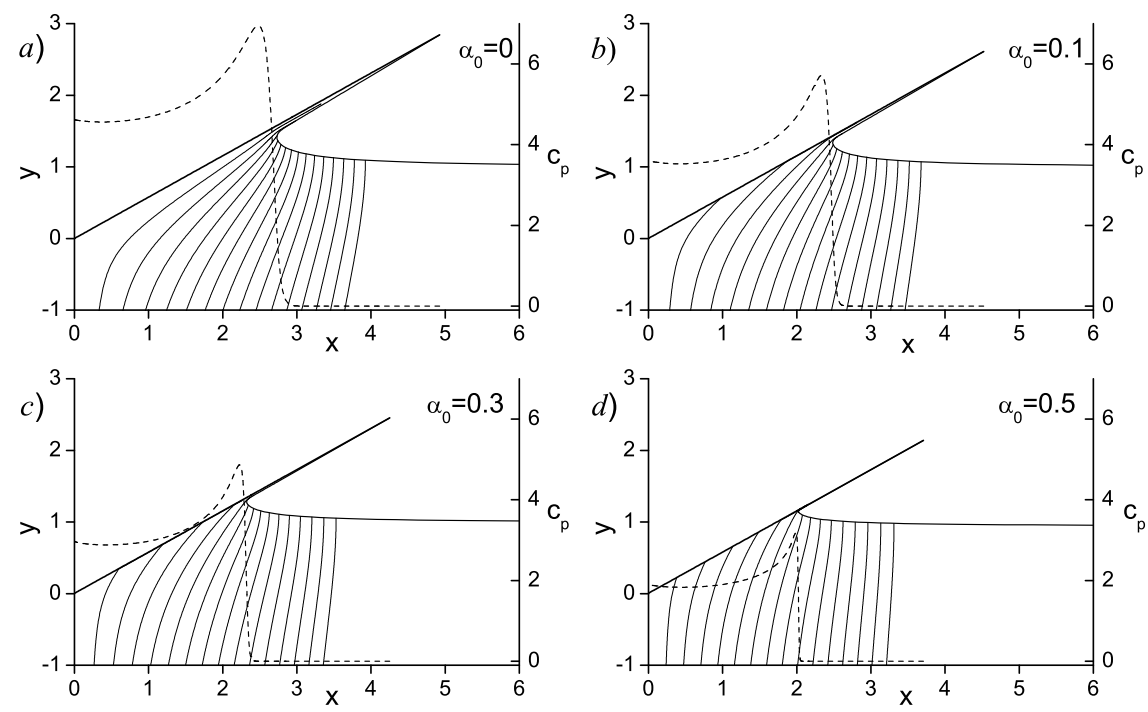

Figure 3: (a) Streamlines with $\Delta \psi=0.2$ and the free surface shape (solid lines), and the pressure distribution (dashed lines) along the porous surface for $\alpha_{A}=60^{\circ}$ and $\alpha=90^{\circ}:(a)$ $\alpha_{0}=0 ;(b) \alpha_{0}=0.1 ;(c) \alpha_{0}=0.3$ and $(d) \alpha_{0}=0.5$.

Further results for streamline patterns, the free surface shape and the pressure distributions for the case in Table 1 are given in figure 3. As discussed by $\mathrm{Wu} \&$ Sun [13], when a fluid particle moves towards an impermeable body surface, its path is blocked and has to make a sharp turn to move along the body surface. This leads to a large acceleration and therefore large pressure gradient near the jet root, as shown in figure $3 a$. When the body surface is permeable, the blockage to path of the fluid flow is less solid. The peak pressure near the jet root decreases. This decrease continues as the permeability coefficient $\alpha_{0}$ increases. This makes the difference between the peak pressure and the ambient pressure on the other side of the body or insider the jet much smaller. We notice that when $\alpha_{0}$ increases, more liquid will move through the body surface, which can be reflected by $v_{n}=\alpha_{0} c_{p}$. In fact we can let the stream function $\psi=0$ on the symmetry line or the $y$-axis. With the same increment $\Delta \psi=0.2$ in figures $3 a$ to $3 d$, the number of streamlines intersecting the body surface from the tip of the wedge to the jet root is approximately equal to the flux rate into the 

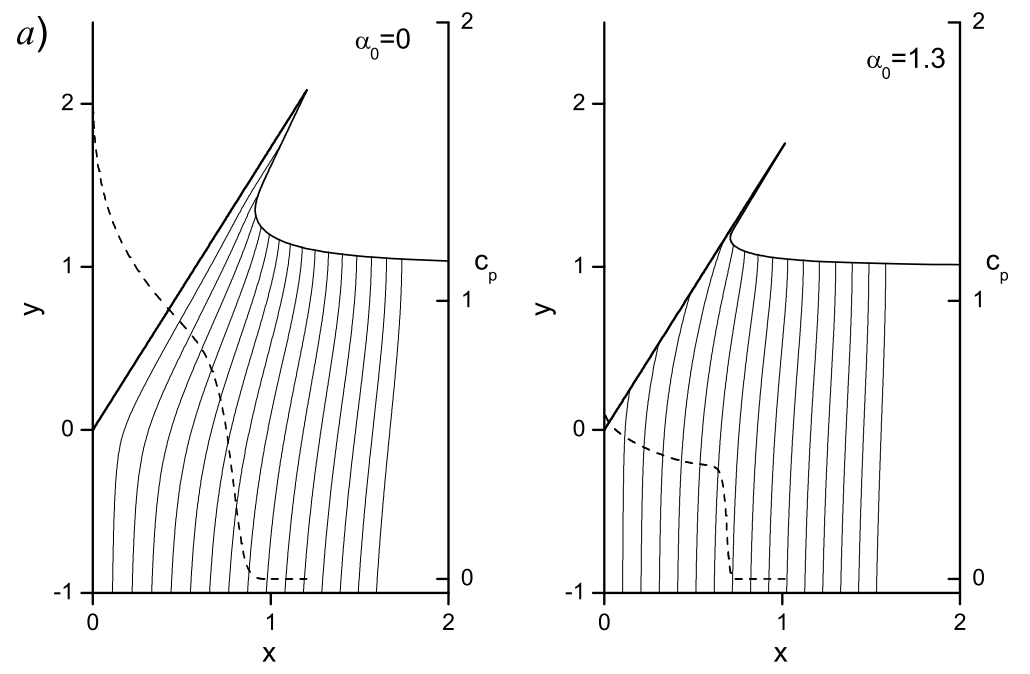

Figure 4: (a) Streamline pattern with $\Delta \psi=0.1$ and the free surface shape (solid lines), and the pressure distribution (dashed lines) along the porous surface for $\alpha_{A}=30^{\circ}$ and $\alpha=90^{\circ}$ : (a) $\alpha_{0}=0 ;(b) \alpha_{0}=1.3$.

body surface. It is evident that this rate increases with increase of $\alpha_{0}$. When more liquid passes through the body surface, the position of the jet root moves towards the $y$-axis. The jet becomes thinner and shorter, and the contact angle $\mu$ becomes smaller. When $\alpha_{0} \rightarrow \infty$, Eq.(24) gives $c_{p} \rightarrow 0$ to provide the a finite value of $v_{n}$. This means that the wetted solid surface has become a free surface. The velocity field in the whole flow region becomes undisturbed. The wetted length of the solid wall tends to the distance between tip of the body and its intersection with the undisturbed liquid wedge.

For $\alpha_{A}=30^{\circ}$ the results for streamline patterns, the free surface shape and the pressure distributions are shown in figure 4 . At $\alpha_{0}=0$ the pressure on the wedge side is much lower than that for $\alpha_{A}=60^{\circ}$, and the pressure peak near the root of the splash jet in figure 3 is absent. This agrees with previous studies of water impact problems [14]. It leads to a smaller normal component of the velocity according to Eq. (24) and, consequently, smaller flow rate through the porous surface of the wedge. 
Although the permeability coefficient $\alpha_{0}$ is larger than for the case $\alpha_{A}=60^{\circ}$ in figure 3 , the flowrate trough the permeable surface corresponding to the $4^{\text {th }}$ streamline, crossing the wedge side in figure $4 b$, is $q=4 \Delta \psi=0.4$, or about $70 \%$ of the total flowrate $Q=v \tan 30^{\circ} \approx 0.58$ trough the cross-section of the solid wedge and the undisturbed free surface. Here, $\Delta \psi=0.1$ is the increment of the stream function between two neighbour streamlines in figure 4 . Thus, although the $\alpha_{0}$ is about 2.6 times larger for the case shown in figure $4 b$, the relative flowrate trough the permeable wedge side is approximately the same as for the case $\alpha_{A}=60^{\circ}$ in figure $3 d$. This occurs due to the lower pressure on the permeable wedge side for $\alpha_{A}=60^{\circ}$ and $\alpha_{0}=0$.

\subsection{Impact of the liquid wedge on the perforated body.}

Perforated body is used widely in coastal and offshore engineering $[1,5,6]$ as a mean to decrease the contact area by allowing the part of the liquid to flow through the body surface. This also reduces a high-pressure peak and the total force during wave impact. Iafrati, Miloh \& Korobkin [7] considered the liquid impact caused a perforated block sliding into the water along a sloping beach. Because the gravity effect is ignored, we can rotate the beach in their case to become the $y$-axis and the problem then becomes the same as that shown in figure 1a. Besides, Iafrati \& Korobkin [6] also considered a perforated wedge entering into the calm free surface. In both cases, the permeability of the body surface is characterized by the coefficient $\chi_{0}$ in Eq. (25). Another model for perforated structures has been proposed by Cooker [15]. He considered a perforated wall as a cascade of impermeable bodies with some gap between them, through which the liquid passes in the form of free streamline jets. Such a model gives the possibility to determine the coefficient $\chi_{0}$. However this is only for some simple geometries of the wave-breaker structures.

Figure 5 show the results for impact of a liquid wedge hitting on a perforated flat wall. Provided are the streamlines, free surface and the pressure distributions along the impermeable wall and a perforated wall with $\chi=0.5$. The angle of the liquid wedge has been taken as $\alpha=10^{\circ}, \alpha=30^{\circ}$ and $\alpha=60^{\circ}$. 

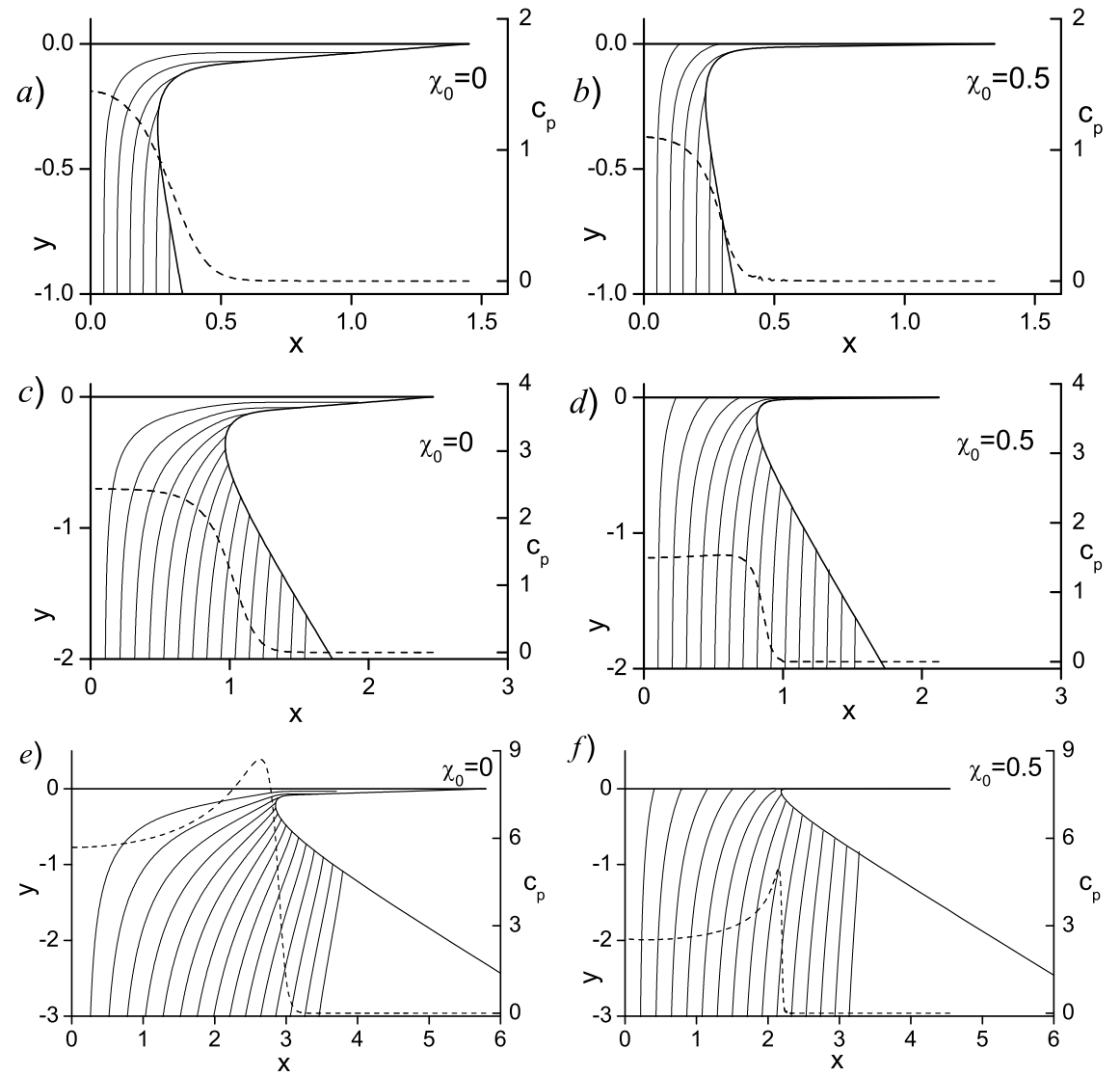

Figure 5: (a) Streamline pattern, free surface (solid lines), and the pressure distribution (dashed lines) along a flat wall: $\alpha=10^{\circ},(a) \chi_{0}=0$ and $(b) \chi_{0}=0.5 ; \alpha=30^{\circ},(c) \chi_{0}=0$ and $(d) \chi_{0}=0.5 ; \alpha=60^{\circ},(e) \chi_{0}=0$ and $(f) \chi_{0}=0.5$.

As expected, the pressure on the wall will decrease when it is perforated. The reduction of the pressure is more pronounced at the locations where the corresponding pressure on the impermeable wall is higher. This is because at higher pressure more liquid moves into the wall as it can be seen from Eq. (25). The larger pressure is, the bigger pressure reduction occurs. We also notice that the jet root moves towards the centre of the impact for the perforated surface. This effectively reduces the area affected by the hydrodynamic pressure. The tip of the jet also moves towards the centre and the jet itself becomes thinner.

In figure 6 the flow configuration, streamlines and pressure distribution are 

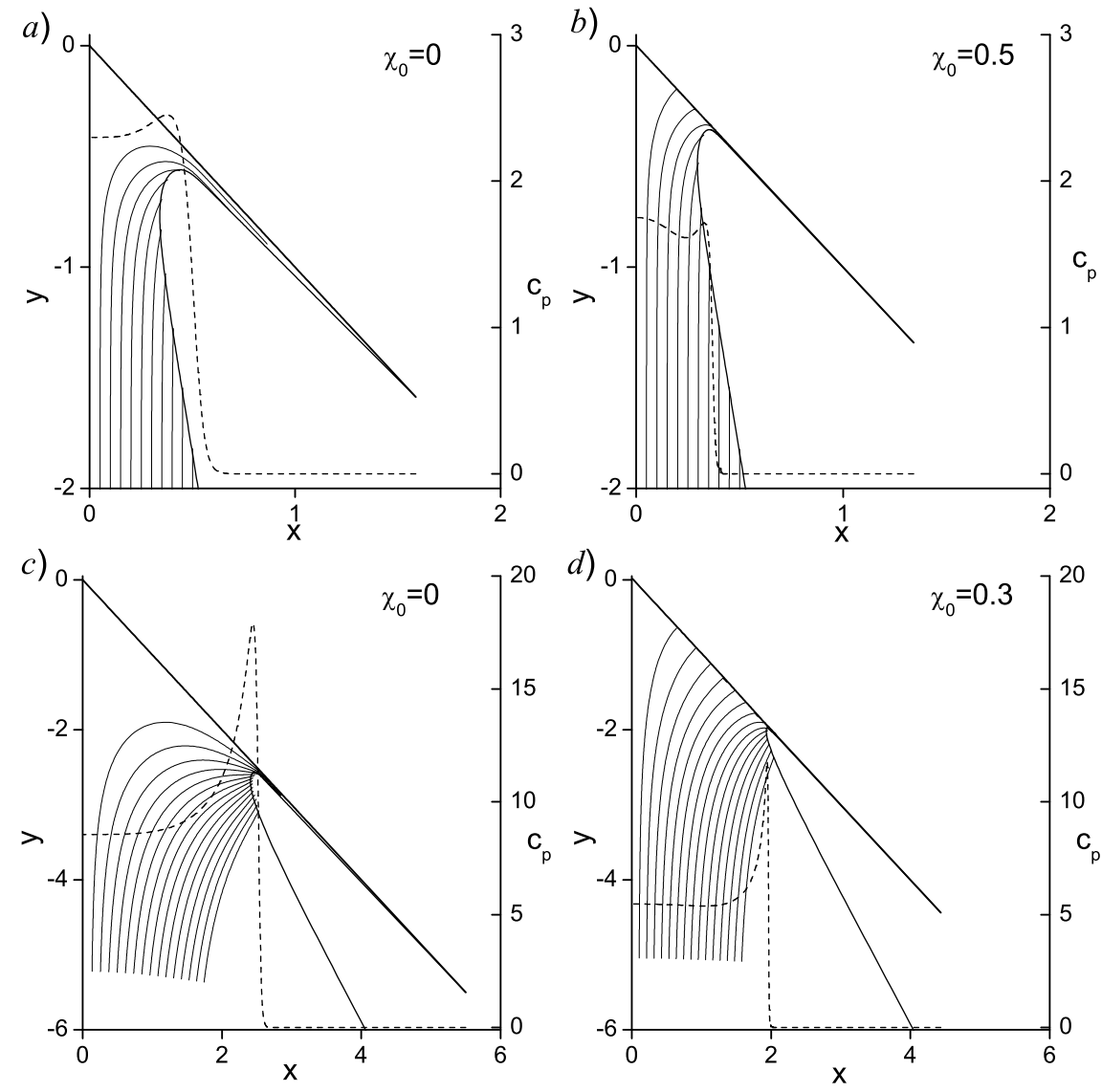

Figure 6: (a) Streamline patterns, free surfaces (solid lines), and the pressure distribution for impact of liquid and solid wedges with $\alpha_{A}=135^{\circ}: \alpha=10^{\circ}, \Delta \psi=0.05$ (a) $\chi_{0}=0$ and (b) $\chi_{0}=0.5 ; \alpha=30^{\circ}, \Delta \psi=0.05$ (c) $\chi_{0}=0$ and $(d) \chi_{0}=0.3$. 


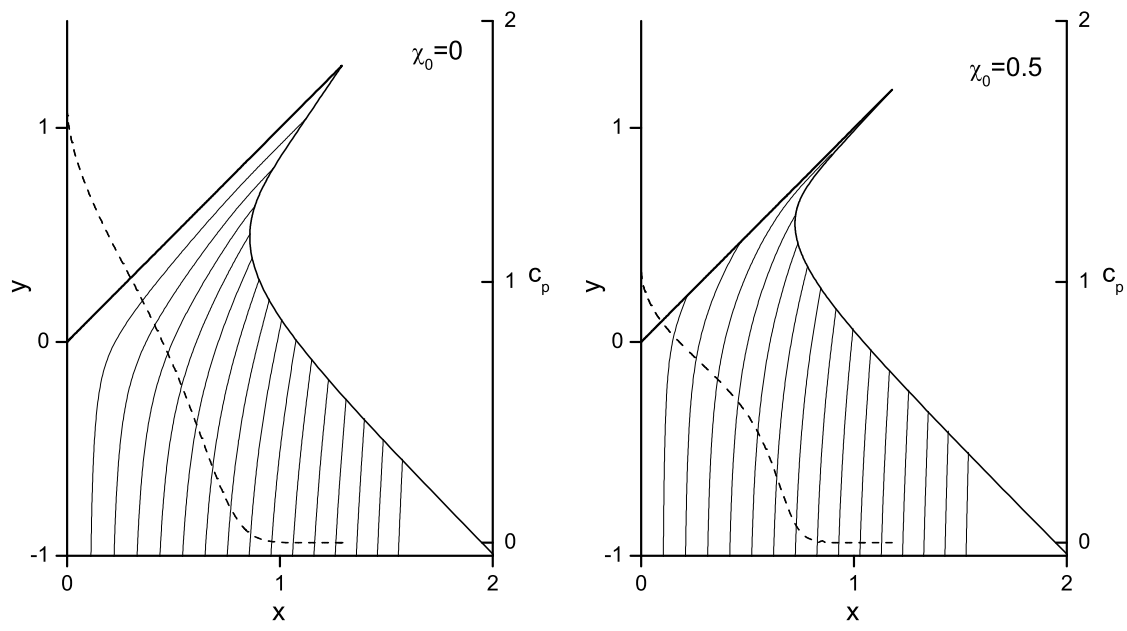

Figure 7: (a) Streamline patterns, free surfaces (solid lines), and the pressure distribution for the liquid and solid wedges $\alpha_{A}=45^{\circ}$ and $\alpha=45^{\circ}$, increment in the stream function $\Delta \psi=0.1$, and $(a) \chi_{0}=0$ and $(b) \chi_{0}=1.0$.

shown for the two deadrise angles $\Delta_{f}$, which is the angle between the solid surface and the undisturbed liquid surface, $\Delta=180^{\circ}-\alpha-\alpha_{A}=35^{\circ}$ for cases $(a)$ and $(b)$, and $\Delta_{f}=15^{\circ}$ for $(c)$ and $(d)$. For cases $(a)$ and $(c), \chi=0$, the sparse distribution of the streamlines near the apex of the solid wedge indicates the low-velocity, or almost a stagnation region. For $\Delta_{f}=35^{\circ}$ the low-velocity region also occurs near the apex of the solid wedge. The tip jet, or the jet attached on the body surface, becomes very thin at $\chi=0.5$. At even larger $\chi$, the jet will be even thinner, which would cause computational difficulty. Because of that at $\Delta_{f}=15^{\circ}$ the maxima $\chi$ for which the converged results are obtained is 0.3 .

Comparing the results in figures 5 and 6 we can see that the smaller deadrise angle causes the larger pressure peak near the root of the tip jet as well as the larger pressure on the entire wedge surface. However, larger permeability of the solid wedge leads to larger reduction of the high pressure due to the larger flowrate through the wedge surface for both linear (Eq. (24)) and quadratic (Eq. (25)) relationships between the normal velocity and the pressure. 
Results for the angles of the liquid and solid wedges $\alpha_{A}=\alpha=45^{\circ}$ are shown in Figure 7. For the impermeable surface in case $(a)$, the pressure decreases almost linearly from the wedge apex to the root of the tip jet, while in case $(b)$ the pressure decreases more mildly and then faster near the root of the tip jet. This is caused by larger pressure reduction near the apex of the wedge due to the larger flowrate into the wedge side there.

\section{Conclusions}

We have developed a method which generalizes previous water-impact studies and accounts for certain essential features associated with a permeability of the solid body. The focus is on some configuration in which the flow can be treated as self similar at earlier stage of the impact. The mathematical approach is based on the integral hodograph method, which enables the original problem in a physical domain to be reduced to a system of integro-differential equations along the straight lines. These equations are solved numerically through the method of successive approximation. The numerical procedure has been verified by convergence study and comparison with some known results.

The presented calculations confirmed the reduction of the hydrodynamic pressure for a porous or perforated wedge entering the flat free surface and revealed the similar effect for a liquid wedge impacting a permeable wedge. This effect magnifies in the case of smaller deadrise angle between the side of the solid and the undisturbed liquid surface. The permeability of the body also affects the tip jet which becomes a much thinner liquid film on the body surface even at moderate permeability condition.

\section{Acknowledgements}

This work is supported by Lloyds Register Foundation (LRF) through the joint centre involving University College London, Shanghai Jiaotong University and Harbin Engineering University, to which the authors are most grateful. LRF 
supports the advancement of engineering-related education, and funds research and development that enhances safety of life at sea, on land and in the air.

\section{References}

[1] B. Molin, Motion Damping by Slotted Structures. In Hydrodynamics: Computations, Model Tests and Reality, Developments in Marine Technology. Elsevier, 1992.

[2] S.D. Howison, J.R. Ockendon and S.K. Wilson. Incompressible water entry problems at small deadrise angles. J. Fluid Mech. 222(1991), 215-230.

[3] J.M. Oliver Second-order Wagner theory for two-dimensional water-entry problems at small deadrise angles. J. Fluid Mech. 572(2007), 59-85.

[4] M. Reinhard, A.A. Korobkin, M. J. Cooker. Water entry of a flat elastic plate at high horizontal speed. J. Fluid Mech. 724(2013), 123 - 153.

[5] B. Molin A.A. Korobkin. Water entry of perforated wedge. In 16th IWWWFB Proc. (Eds. K. Mori and H. Iwashita), Hiroshima, 22-25 April (2001), 121- 124.

[6] A. Iafrati, A.A. Korobkin Self-similar solutions for porous/perforated wedge entry problem. In 20th IWWWFB Proc. Oslo, 29 May - 1 June (2005).

[7] A. Iafrati, T. Miloh, A.A. Korobkin. Oblique water entry of a block sliding along a sloping beach. Proceedings of International Conference on Violent Flows, Fukuoka, Japan (2007).

[8] Y.A. Semenov, A. Iafrati. On the nonlinear water entry problem of asymmetric wedges. J. Fluid Mech., 547(2006), 231 - 256.

[9] Y.A. Semenov, L.J. Cummings. Free boundary Darcy flows with surface tension: analytical and numerical study. Euro. J. Appl. Math., 17(2006), $607-631$. 
[10] Y.A. Semenov, G.X. Wu Asymmetric impact between liquid and solid wedges. Proc. R. Soc. A 469(2013).

[11] Y.A. Semenov, G.X. Wu, J.M. Oliver. Splash Jet Caused by Collision of Two Liquid Wedges. J. of Fluid Mech. 737(2013), 132 - 145.

[12] Z. Huang, Y. Li, Y. Liu. Hydraulic performance and wave loadings of perforated/slotted coastal structures: a review. Ocean Engn. 38(2011), 10311053.

[13] G.X. Wu, S.L. Sun. Similarity solution for oblique water entry of an expanding paraboloid. J. Fluid Mech. 745(2014), 398.

[14] Z.N. Dobrovol'skaya. On Some problems of similarity flow of fluid with a free surface. J. Fluid Mech. 36(1969), 805 - 829.

[15] M. Cooker. A theory for the impact of a wave breaking onto a permeable barrier with jet generation. J. Eng. Math 79(2013):1-12. 